
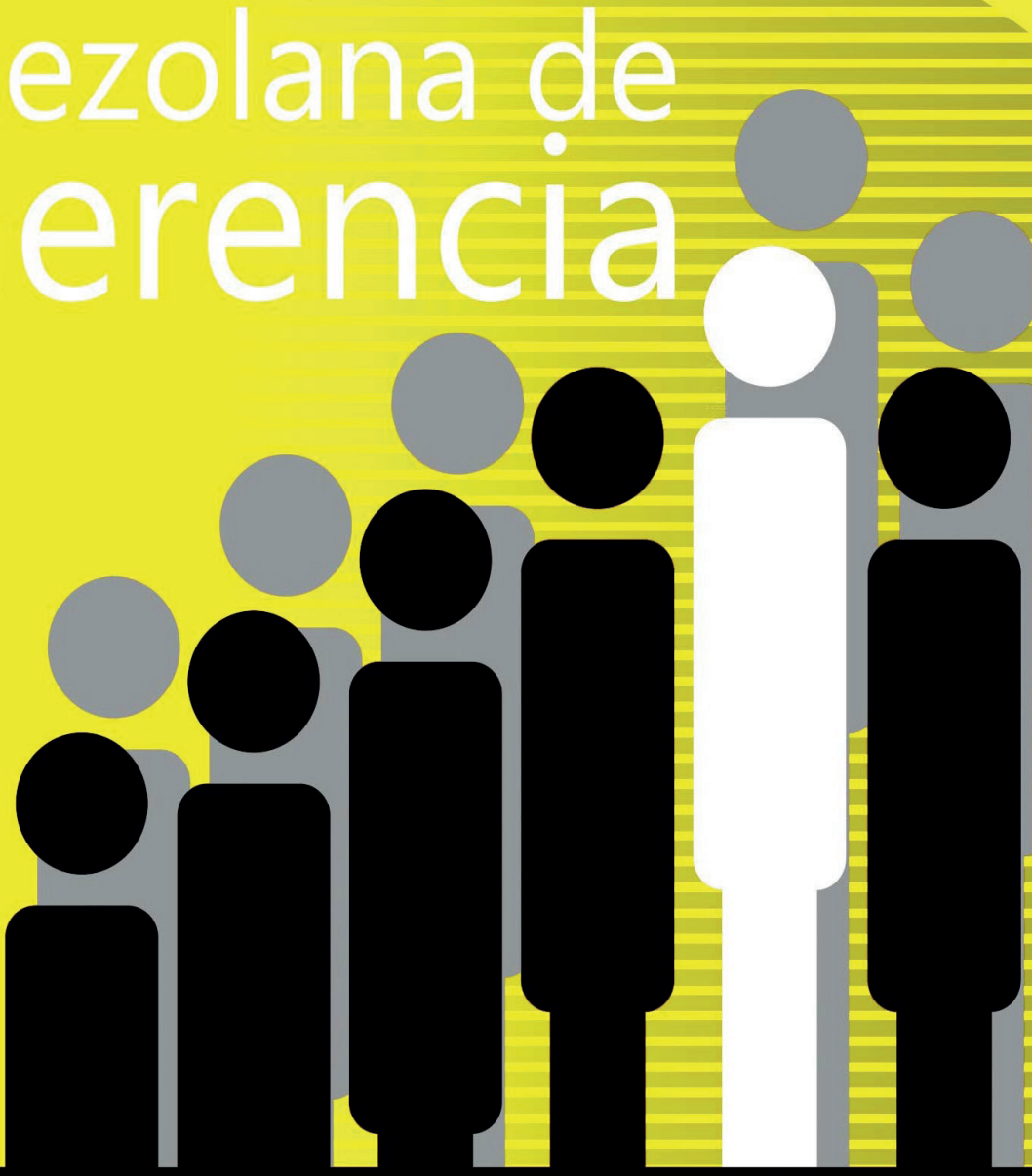


\title{
Configuraciones de Prácticas en la Gestión del Capital Humano y Resultados Organizativos
}

\author{
Medina Lorza, Aida* \\ Acosta Naranjo, Alejandro* \\ Revuelto Taboada, Lorenzo ${ }^{* \star *}$
}

\section{Resumen}

A pesar de la creciente importancia que se le ha venido dando al capital humano como objeto de investigación en el ámbito organizacional, en la actualidad sigue habiendo disenso sobre los efectos del uso de configuraciones de prácticas en su gestión sobre los resultados organizativos. Por esto, el presente estudio tiene como objetivo principal analizar los efectos de la adopción de configuraciones de prácticas en la gestión del capital humano sobre los resultados organizativos. Para este fin, se hace una revisión de literatura que posibilita la formulación de hipótesis. Asimismo, se distribuyeron 550 cuestionarios auto administrado a una muestra de 50 empresas nacionales colombianas, multinacionales colombianas, multinacionales norteamericanas y multinacionales españolas, con domicilio todas ellas en Colombia. Los datos se han analizado mediante el análisis por mínimos cuadrados parciales PLS-SEM o PLS path modelling. Los resultados obtenidos sugieren que la utilización de configuraciones de prácticas en la gestión del capital humano no tienen efectos positivos sobre los resultados organizativos. Se concluye que para futuros estudios se deben considerar variables contextuales moderadoras y entender dichas configuraciones de prácticas en el marco global del sistema de gestión humana para cada organización.

Palabras clave: prácticas en la gestión del capital humano; resultados organizativos; modelo de Lepak y Snell.

\section{Recibido: 20.10.20 Aceptado: 15.02.21}

Doctora en dirección de empresas (Universidad de Valencia). Profesora tiempo completo del departamento de gestión organizacional. Facultad de Ciencias Administrativas y Económicas. Universidad Icesi. Colombia. E-mail: afmedina@icesi.edu.co ORCID: https://orcid.org/0000-0002-3918-6821

* Doctor en dirección de empresas (Universidad de Valencia). Profesor tiempo completo del departamento de gestión organizacional. Facultad de Ciencias Administrativas y Económicas. Universidad Icesi. Colombia. E-mail: aacosta@icesi.edu.co ORCID: https://orcid.org/0000-0003-1735-4166

*** Doctor en ciencias económicas (Universidad de Valencia). Profesor titular del departamento de dirección de empresas “Juan José Renau Piqueras”. Facultad de Economía. Universidad de Valencia. España. E-mail: lorenzo.revuelto@uv.es ORCID: https://orcid.org/0000-0002-4957-8626 


\title{
Practice Configurations in Human Capital Management and Organizational Results
}

\begin{abstract}
Despite the growing importance that has been given to human capital as an object of research in the organizational field, at present there is still disagreement about the effects of the use of configurations of practices in its management on organizational results. For this reason, the main objective of this study is to analyze the effects of the adoption of practice configurations in human capital management on organizational results. For this purpose, a literature review is made that enables the formulation of hypotheses. Likewise, 550 self-administered questionnaires were distributed to a sample of 50 Colombian national companies, Colombian multinationals, North American multinationals and Spanish multinationals, all of them domiciled in Colombia. The data have been analyzed using the partial least squares analysis PLS-SEM or PLS path modeling. The results obtained suggest that the use of practice configurations in human capital management do not have positive effects on organizational results. It is concluded that for future studies, moderating contextual variables should be considered and said configurations of practices should be understood in the global framework of the human management system for each organization.
\end{abstract}

Keywords: practices in human capital management; organizational results; Lepak and Snell model.

\section{Introducción}

Aunque la investigación sobre la "gestión del capital humano" (GCH) es cada vez más rigurosa, aún no existe un consenso sobre los efectos de la utilización de configuraciones de prácticas en los resultados organizativos (Boon, Den Hartog \& Lepak, 2019). Según Pham (2020), los efectos son positivos. En este sentido, Lepak \& Snell (1999-2002) refieren que un mayor grado de congruencia en las "configuraciones de prácticas en la gestión del capital humano" ( $\mathrm{CPCH})$ debería estar asociado a lograr mejores resultados organizativos. Melián \& Verano (2006,
2008), indican que no todas las empresas agrupan a su capital humano según las combinaciones de valor y singularidad propuestas por Lepak \& Snell (1999). Delery \& Gupta (2016), Gorman et al, (2017), recomiendan sistemas de prácticas únicas para la totalidad de los miembros de la organización sin tener en cuenta las peculiaridades de los diferentes colectivos de empleados a los que deben ser aplicadas.

Desde una perspectiva dinámica, Buller \& McEvoy, (2012) plantean que factores contextuales pueden requerir configuraciones particulares para cada firma. En este sentido, Andersén (2019) expone que la orientación empresarial 
modera la relación entre el capital humano de la empresa y el desempeño de la misma.

Por lo tanto, en consonancia con Melian \& Verano (2008), se considera que el estudio de las $\mathrm{CPCH}$, proporciona una mejor comprensión sobre su impacto en los resultados organizativos.

Por todo lo anterior, el presente estudio encuentra justificación al aportar resultados empíricos a la discusión teórica sobre la validez o no de los efectos positivos de la utilización de las $\mathrm{CPCH}$ sobre los resultados organizativos. Así mismo se justifica al proporcionar evidencias que contribuyen a la toma de decisiones estratégicas de quienes son responsables por la $\mathrm{GCH}$ en las organizaciones. Por ello, este estudio tiene como finalidad dar respuesta a la siguiente pregunta de investigación: ¿cuáles son los efectos de la utilización de configuraciones de prácticas en la gestión del capital humano sobre los resultados organizativos?

Para tal fin, se ha adoptado como marco de referencia teórico el modelo de configuraciones de prácticas propuesto por Lepak \& Snell (1999-2002). Así mismo, se ha considerado para el abordaje de los resultados organizativos la predisposición a abandonar la empresa (Intention To Quit) utilizada por Siong et al, (2006); la productividad laboral que muestra un resultado más "cercano" a los empleados que la rentabilidad (Black \& Linch, 2004); el ROA o rentabilidad económica (Walsh, 1994: 60) y, finalmente el ROE o rentabilidad financiera o "rendimiento que los proveedores de capital riesgo obtienen" (Walsh, 1994: 58).

Se obtuvo una muestra de 550 sujetos pertenecientes a 20 empresas nacionales colombianas,
10 multinacionales colombianas, 10 multinacionales norteamericanas y 10 multinacionales españolas. Todas ellas con $\mathrm{CPCH}$.

En cuanto a la capacidad predictiva del modelo estructural, se analizó la $\mathrm{R}^{2}$. Se examinó los coeficientes $\beta i j$ en su relación con cada variable independiente y la dependiente. Así mismo, mediante el programa PLS (bootstraping) se determinó la significancia de los coeficientes $\beta \mathrm{ij}$ y las cargas factoriales (outer loadings) a partir los valores estadísticos $\mathrm{T}$.

Los resultados obtenidos parecen indicar que utilizar configuraciones de prácticas puede conllevar más inconvenientes que ventajas. La incidencia del nivel de ajuste en los resultados se ha mostrado irrelevante y, en muchos casos la influencia detectada ha sido de signo contrario a lo esperado al tomar como referente las hipótesis implícitas en el modelo de Lepak \& Snell (1999-2002).

\section{Estimaciones teóricas sobre el modelo de configuraciones de prácticas de Lepak \& Snell}

El modelo de Lepak \& Snell (1999-permite relacionar características esenciales del capital humano (valor y singularidad), con cuatro conjuntos de prácticas en la gestión de este. Para los autores, la idoneidad de las prácticas depende del valor que el capital humano consecuente aporta a la organización. Al superponer las dimensiones de valor y de singularidad del capital humano, se puede definir un modelo compuesto por cuatro cuadrantes que vinculan las diferentes combinaciones de $\mathrm{CPCH}$ (diagrama1). 


\section{Diagrama 1 \\ Sumario de configuraciones de prácticas en la gestión del capital humano}

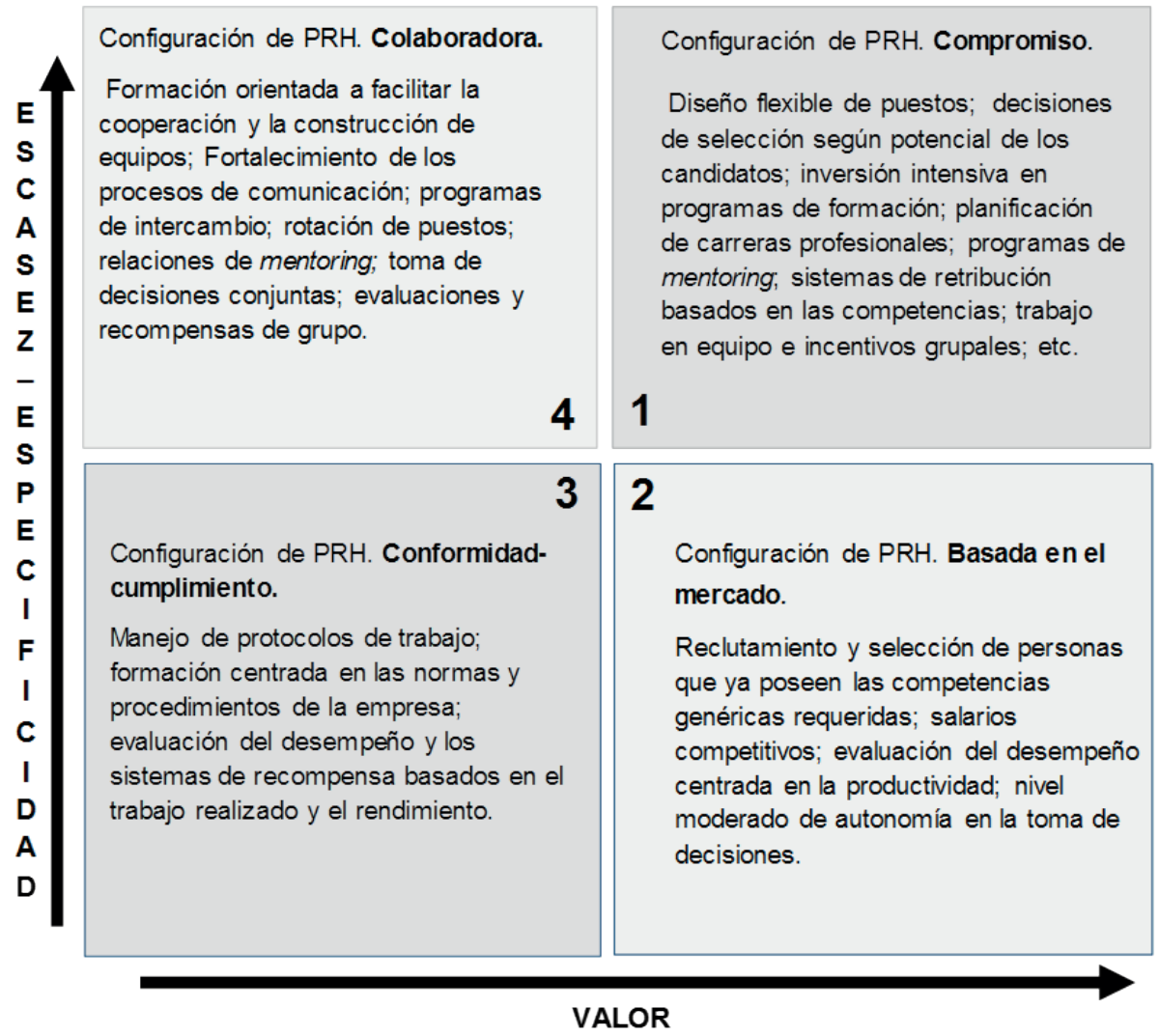

Fuente: Adaptado de Lepak \& Snell (1999-2002)

Cuadrante 1. Este primer cuadrante corresponde al capital humano singular o específico y que aporta mucho valor a la organización. La CPCH tendiente al compromiso propuesta por Lepak \& Snell (1999) es muy similar a los sistemas de prácticas de alto compromiso o desempeño propuestos por Pfeffer (1994). A modo de ejemplo Lepak \& Snell (1999) señalan como prácticas para fortalecer el compromiso las siguientes: diseño flexible de puestos de trabajo, para facilitar el cambio y la adaptación; basar las decisiones de selección en la evaluación del potencial de los candidatos, en lugar de simplemente en los conocimientos y las habilidades actuales; la inversión intensiva en programas de formación; la planificación de carreras profesionales; 
los programas de mentoring; los sistemas de retribución basados en las competencias; el trabajo en equipo y los incentivos grupales; sistemas de evaluación del desempeño centrados en el desarrollo; etc.

Cuadrante 2. En este caso se trata de capital humano que si bien es valioso, por su baja especificidad puede encontrarse disponible en el mercado laboral. La CPCH propuesta por Lepak \& Snell (1999-2002), estará en este caso basada en el mercado. Para Lepak \& Snell (1999-2002), basados en autores como Koch \& McGrath (1996) y Miles \& Snow (1984), el reclutamiento y selección de personal tendrá como criterio primordial que los candidatos dispongan de las competencias genéricas requeridas para su rápida utilización. Adicionalmente, las empresas deberán ofrecer salarios competitivos (garantizar la equidad externa) para poder adquirir este capital humano valioso pero genérico $\mathrm{y}$, se centrará en la productividad a la hora de evaluar el desempeño de los empleados contratados. De igual manera, las empresas deberán garantizar que los empleados puedan gozar de cierto nivel de autonomía a la hora de tomar decisiones que pudieren impactar positivamente en el valor (Snell \& Dean, 1994).

Cuadrante 3. Este cuadrante corresponde a capital humano genérico y de poco valor estratégico para la empresa. Para Lepak \& Snell (19992002) la $\mathrm{CPCH}$ en este caso estará fundamentada en el cumplimiento o conformidad. De acuerdo con los autores, las empresas para garantizar el cumplimiento, se enfocan en lograr que se cumplan las normas, parámetros y políticas institucionales relacionadas con el quehacer laboral.

Lepak \& Snell (1999-2002) plantean, en referencia a esta $\mathrm{CPCH}$, basados en autores como Becker (1964) y Rousseau \& Parks (1993), que difícilmente las empresas invertirán en la formación y el desarrollo de este tipo de $\mathrm{RRHH}$ y, cuando lo hagan, la formación se centrará en las normas y procedimientos de la empresa. Así mismo, con base en autores como Mahoney (1989) y Snell \& Dean (1994), plantean que la evaluación del desempeño y los sistemas de recompensa estarán basados en el rendimiento del trabajo realizado y, en los sistemas de evaluación y recompensa definidos para situaciones de agencia (Eisenhardt, 1988).

Cuadrante 4. Finalmente, este cuadrante corresponde a capital humano singular pero con valor estratégico poco relevante. La CPCH que corresponde a este cuadrante es la que Lepak \& Snell (1999) denominan colaborativa. Dado que, generalmente, es necesario que los empleados de los distintos asociados trabajen conjuntamente para conseguir sinergias, las prácticas en esta $\mathrm{CPCH}$ deben fomentar la cooperación y que se comparta información.

Lepak \& Snell (1999-2002) establecen, soportados en autores como Dyer (1996); Nonaka \& Takeuchi (1995); Quinn et al, (1996), que las empresas difícilmente dedicarán recursos para formar y desarrollar a sus asociados, pero sí invertirán en la relación y su efectivo funcionamiento. La formación irá, por tanto, orientada a facilitar la cooperación y construir equipos. Es probable que se invierta en procesos de comunicación, programas de intercambio, rotación de puestos, relaciones de mentoring, para facilitar el que se comparta información así como la transferencia de conocimiento necesaria para la toma de decisiones conjuntas. Asimismo es posible que se 
utilicen evaluaciones y recompensas de grupo, para animar a los empleados de los diferentes asociados a compartir y transferir información.

\section{Configuraciones de prácticas en la gestión del capital humano de Lepak y Snell y los resultados organizativos}

Lepak \& Snell (1999-2002) señalan que es posible combinar los argumentos previamente analizados para los cuatro cuadrantes $y$, que ello puede ofrecer una perspectiva más completa de cómo los gerentes podrían tomar las decisiones en relación a las prácticas en la $\mathrm{GCH}$ a utilizar. En su modelo los autores proponen que las elecciones sobre la $\mathrm{CPCH}$ a utilizar dependen del potencial de creación de valor de las competencias de los empleados y de su singularidad (escasez/especificidad) respecto a una empresa en particular. $A$ este respecto, Pham (2020) y Wuen et al, (2020), concluye que las prácticas en la $\mathrm{GCH}$ tienen un efecto positivo en los resultados de los negocios.

De acuerdo a Lepak \& Snell (19992002) un mayor grado de congruencia en las $\mathrm{CPCH}$ en las empresas debería estar asociado con el logro de mejores resultados organizativos. Por tanto, se propone la siguiente hipótesis:

H1. La aplicación de configuraciones de prácticas en la gestión del capital humano tiene un efecto positivo sobre los resultados organizativos.

De otro lado, las evidencias empíricas acumuladas en relación con la propuesta de Lepak \& Snell (19992002) son todavía escasas y no del todo concluyentes. En el trabajo de
Lepak et al, (2003), si bien se indica que los patrones de utilización de las diferentes configuraciones de prácticas están significativamente relacionados con los resultados empresariales, solo abordan "parte" del modelo "ideal" planteado en Lepak \& Snell (19992002). Adicionalmente, los resultados obtenidos en los trabajos de Melián \& Verano (2006, 2008), indican que no todas las empresas agrupan a su capital humano según las cuatro combinaciones de valor y singularidad propuestas por Lepak \& Snell (1999-2002).

Desde una perspectiva dinámica, Buller \& McEvoy (2012) anotan que factores contextuales pueden requerir $\mathrm{CPCH}$ particulares para cada firma. Sus resultados revelan variaciones asociadas al valor y la especificidad del capital humano en las $\mathrm{CPCH}$ dentro de las empresas. Concluyen los autores que la efectividad de las $\mathrm{CPCH}$ solo se podrá evidenciar si estas son congruentes con la estrategia empresarial. En este sentido, Liu et al, (2007) condicionan el impacto del capital humano en el rendimiento organizativo a que las prácticas y sistemas de gestión apoyen la estrategia comercial de una empresa. Andersén (2019) plantea que la orientación empresarial modera la relación entre el capital humano específicos de la empresa y el desempeño de la empresa. Y, Huo et al, (2016) y Crandall \& Crandall (2008) argumentan que la incidencia del capital humano en los resultados organizativos es indirecta. Manifiestan los autores que el capital humano mediado por diferentes configuraciones de prácticas en su gestión, afecta de manera directa la eficiencia y eficacia de la operación empresarial, lo cual, a su vez, afecta los resultados organizativos.

Con base en la variedad de 
argumentos esbozados anteriormente sobre la afectación no directa de las $\mathrm{CPCH}$ sobre los resultados organizativos, contraria a lo propuesto por Lepak \& Snell (1999-2002), se propone la siguiente hipótesis nula:

Ho. La aplicación de configuraciones de prácticas en la gestión del capital humano no tiene un efecto positivo sobre los resultados organizativos.

Ahora bien, basados en la Teoría del Capital Humano (Becker, 1964), Lepak \& Snell (1999-2002) argumentan que las empresas se protegen evitando transferir sus inversiones en capital humano a otras empresas, lo cual, las lleva a implementar configuraciones de prácticas cuyo propósito es el de atraer, retener y motivar ese "capital humano" para desarrollar ventajas competitivas y alcanzar sus objetivos estratégicos. En este sentido, Fayad \& Easa (2020) plantean que la implementación adecuada de las $\mathrm{PCH}$ influye en el compromiso de los empleados y estos a su vez afectan positivamente los resultados de la empresa. En vista de los argumentos anteriores, se formula la siguiente hipótesis:

H1.1. La aplicación de configuraciones de prácticas en la gestión del capital humano tiene una influencia negativa sobre la predisposición a abandonar la empresa (Intention To Quit).

Desde la teoría de recursos y capacidades o teoría de la empresa basada en los recursos (Barney, 19861991), Lepak \& Snell (1999-2002) argumentan que las empresas deberían tomar sus decisiones en relación a la adopción de $\mathrm{CPCH}$, teniendo en cuenta en qué medida los empleados aportan conocimientos, habilidades y destrezas, que pueden contribuir a sostener o mejorar las competencias clave de las mismas (Quinn, 1992). De esta manera, las empresas invierten recursos para mejorar las capacidades de los empleados únicamente cuando las inversiones realizadas se pueden recuperar merced a mejoras en la productividad de los mismos (Tsang et al, 1991). Por lo tanto, se propone la siguiente hipótesis:

H1.2. La aplicación de configuraciones de prácticas en la gestión del capital humano tiene una influencia positiva sobre la productividad laboral.

Por último, Lepak \& Snell (19992002) con base en la Teoría de Costes de Transacción (Williamson, 1975-1981), definen una serie de prácticas en la $\mathrm{GCH}$ que tratan de garantizar el cumplimiento de lo estipulado en el contrato y el logro de resultados empresariales. Estas prácticas son: la retribución variable, la evaluación de los resultados y del comportamiento, la promoción a través de los mercados internos de trabajo, etc., (Mozo \& Pérez, 2001). De acuerdo con esta teoría, las empresas tratan de "adquirir" en el mercado laboral las competencias necesarias para garantizar la rentabilidad de sus negocios y lograr una óptima relación costo beneficio. Es por estos argumentos qué formulamos las siguientes hipótesis:

H1.3. La aplicación de configuraciones de prácticas en la gestión del capital humano tiene una influencia positiva en el ROA o rentabilidad económica.

H1.4. La aplicación de configuraciones de prácticas en la gestión del capital humano tiene una influencia positiva en el ROE o rentabilidad financiera. 


\section{Consideraciones metodológicas de la investigación}

Con la finalidad de responder a las hipótesis de investigación planteadas, se realizó un estudio empírico en empresas nacionales colombianas, multinacionales colombianas $y, \quad$ multinacionales extranjeras con domicilio en Colombia. Se aplicaron cuestionarios, se hizo revisión de documentos sobre estados financieros $y$, entrevistas para aclarar y validar algunos de los resultados obtenidos.

\subsection{Diseño de la Muestra y Recolección de los Datos}

Se obtuvo una muestra compuesta por 550 sujetos pertenecientes a 20 empresas nacionales colombianas, 10 multinacionales colombianas, 10 multinacionales norteamericanas y 10 multinacionales españolas que, tuvieran las mismas características en cuanto a tamaño, poseer un departamento de gestión humana y, utilizar configuraciones de prácticas en la administración del capital humano.

Las empresas se seleccionaron mediante un muestreo aleatorio simple, y contactaron las empresas a través de del DANE$^{1}$ y de ACRIP ${ }^{2}$. El 52\% (26) de estas empresas son empresas de servicios y el $48 \%$ (24) industriales. En lo que respecta a su tamaño, en cuanto a número de empleados promedio, este es de 4.493 empleados.

Para la recolección de los datos, se han utilizado dos cuestionarios diferentes en cada empresa destinados a obtener datos a través de los responsables de la FRH y de los empleados. De esta manera, cada responsable de la función de RRHH en cada una de las empresas, informo sobre el valor y singularidad del capital humano en 10 puestos de trabajo. Posteriormente, cada uno de los empleados pertenecientes a los puestos de trabajo considerado en cada empresa, preciso las prácticas en la GCH de acuerdo a su propia experiencia. De esta manera se pudieron establecer las $\mathrm{CPCH}$ para cada empresa en concordancia con el modelo de Lepak \& Snell (1999-2002).

\subsection{Desarrollo de Medidas}

Para la medición de las configuraciones de prácticas, se utilizó una escala de Likert de 1 (muy en desacuerdo) a 5 (muy de acuerdo) para 56 ítems adaptada de los ítems utilizados por Lepak \& Snell (2002-2003), que fue traducida y ampliada para conseguir un mayor equilibrio en el número de ítems característicos de cada configuración de prácticas.

En cuanto a la medición de los resultados organizativos empleamos los siguientes instrumentos de medida: para Intention to Quit o ITQ los dos ítems trabajados $\mathrm{y}$ validados por autores como Firth et al, (2004) y Siong et al, (2006) y, una escala likert de cinco puntos ( 1 muy pocas veces; 5 muchas veces); para la medición de la productividad laboral se estableció el cociente entre las ventas netas y el 
total de empleados fijos y temporales, utilizado en multitud de trabajos con esta formulación o alguna muy similar (Black \& Linch, 2004). En lo que respecta a la rentabilidad económica o rentabilidad de la inversión, RE Se obtuvo de la razón entre los beneficios antes de intereses e impuestos y el activo total (Walsh, 1994); y, en lo referente a la rentabilidad financiera (RF), o "rendimiento que los proveedores de capital riesgo obtienen" (Westwick, 1997: 64), se obtuvo como el resultado de dividir el beneficio neto después de intereses e impuestos por los fondos propios.

\subsection{Fiabilidad y Validez de las Escalas de Medida}

Para la evaluación de la consistencia interna se utilizó alfa de Cronbach e Índice de Fiabilidad Compuesta IFC. La validez convergente se determinó mediante la comprobación de que las cargas factoriales fuesen mayores a 0,707. La varianza media extraída (AVE) mediante la comprobación de que fuera al menos del $50 \%$. Y, la validez divergente se obtuvo al comparar la raíz del AVE de cada variable latente con los valores de sus correlaciones con las demás variables. Todo ello con base en (Hair et al, 2014).-

En lo que corresponde a la capacidad predictiva del modelo estructural, se analizó la $\mathrm{R}^{2}$. Adicionalmente, se examinó los coeficientes $\beta$ ij en su relación con cada variable independiente y la dependiente. Así mismo, mediante el programa PLS (bootstraping) se determinó la significancia de los coeficientes $\beta \mathrm{ij}$ y las cargas factoriales (outer loadings) a partir los valores estadísticos $\mathrm{T}$.

En general, el porcentaje de varianza explicada en los distintos modelos de las variables de resultados es muy inferior al mínimo recomendado del $10 \%$. Oscila entre un $0,1 \%$ y un $3 \%$. Los valores de los coeficientes $\beta_{i}$ son reducidos y en el caso de la mayoría de las variables de resultados (eficiencia operativa, intención de abandonar la empresa y las variables de rendimiento financiero) contrarios a los esperados y no significativos.

\section{Relación entre las configuraciones de prácticas en la gestión del capital humano y los resultados organizacionales}

Los resultados obtenidos (tabla 1), parecen indicar que utilizar $\mathrm{CPCH}$, puede conllevar más inconvenientes que ventajas. En todo caso la incidencia del nivel de ajuste en los resultados se ha mostrado prácticamente irrelevante y, en muchos casos la influencia detectada ha sido de signo contrario a lo esperado si damos por ciertas las hipótesis implícitas en el modelo de Lepak \& Snell (19992002). 


\section{Tabla 1 \\ Validación de los modelos estructurales. Relación ajuste- resultados}

\begin{tabular}{|c|c|c|c|}
\hline Hipótesis & Descriptivo (Modelo 6: S_ajuste_grados_d) & $\beta$ & t-value \\
\hline $\mathrm{H} 1.1$ & $\begin{array}{l}\text { El ajuste tiene una influencia negativa sobre la Intention } \\
\text { To Quit }\end{array}$ & $-0,14$ & 0,85 \\
\hline $\mathrm{H} 1.2$ & $\begin{array}{l}\text { El ajuste tiene una influencia positiva sobre la productividad } \\
\text { laboral. }\end{array}$ & $-0,20$ & 1,38 \\
\hline $\mathrm{H} 1.3$ & $\begin{array}{l}\text { El ajuste tiene una influencia positiva el ROA o rentabilidad } \\
\text { económica }\end{array}$ & $-0,16^{*}$ & 2.46 \\
\hline $\mathrm{H} 1.4$ & $\begin{array}{l}\text { El ajuste tiene una influencia positiva el ROE o rentabilidad } \\
\text { financiera }\end{array}$ & $-0,17^{* *}$ & 2,96 \\
\hline $\begin{array}{ll}R^{2} & \text { (ITQ): } \\
0,02 & \end{array}$ & Q2 (ITQ): $-0,00$ & & \\
\hline $\mathrm{R}^{2}(\mathrm{PL}): 0,04$ & Q2 (PL): 0,05 & & \\
\hline $\begin{array}{ll}R^{2} & (\mathrm{ROA}): \\
0,03\end{array}$ & Q2 (ROA): 0,03 & & \\
\hline $\begin{array}{ll}\mathrm{R}^{2} & (\mathrm{ROE}): \\
0,03 & \end{array}$ & Q2 (ROE): 0,04 & & \\
\hline
\end{tabular}

Nota: * $p<0,05 ;{ }^{* *} p<0,01$

Fuente: Elaboración propia

Sólo se han detectado relaciones significativas con el ROA y el ROE, pero de signo contrario a lo esperado según los aportes teóricos de la Teoría de Costes de Transacción (Mozo \& Pérez, 2001) al modelo de CPCH de Lepak \& Snell (1999-2002) y, las conclusiones establecidas por Pham (2020) y Wuen et al, (2020) sobre el efecto positivo de las prácticas en la $\mathrm{GCH}$ en los resultados organizativos.

El porcentaje de varianza explicada de las variables de resultados no supera en ninguno de los casos el $7 \%$ y la capacidad predictiva de los modelos es reducida dados los valores de las Q2 que son muy bajos o incluso negativos. Los resultados indican que el uso de configuraciones de prácticas no tiene un efecto positivo sobre la Intention To Quit, la productividad laboral y, la rentabilidad económica y financiera. En consecuencia, los resultados obtenidos indican que no se validan las hipótesis (H1), (H1.1), (H1.2), (H1.3) y (H1.4) y, si se valida la hipótesis nula $(\mathrm{HO})$ en referencia a que la aplicación de $\mathrm{CPCH}$ no tiene un efecto positivo sobre los resultados organizativos.

Ahora bien, como posible 
explicación a esto, se podría pensar en que utilizar configuraciones de prácticas no deben responder a un modelo universal "ideal", sino más bien, a modelos ajustados a las especificidades de cada organización como plantean, desde una perspectiva dinámica, Buller \& McEvoy (2012). Parecería ser que la relación entre las $\mathrm{CPCH}$ y los resultados financieros, económicos y la productividad, está mediada por variables (sustancialmente diferentes para cada organización), de tipo cultural, estratégico, tecnológicos, entre otras. Aspectos no tenidos en cuenta desde los aportes de la teoría de recursos y capacidades (Becker, 1964; Tsang et al, 1991) al modelo de Lepak \& Snell (1999-2002). Otra posible explicación podría hallarse en que en este colectivo de empresas se considere que solo el personal de alto rango posee capital humano valioso (Kehinde, 2012), y por ende, las configuraciones de practica no apliquen en el conjunto de la organización.

De otra parte, al observar los resultados sobre la predisposición a abandonar la empresa o Intention To Quit ITQ, resalta especialmente que las diferencias entre los colectivos más favorecidos o privilegiados y los demás no resulten significativas $y$, en los modelos tengan el signo contrario al esperado según los aportes al modelo propuesto de $\mathrm{CPCH}$ por Lepak \& Snell (1999-2002) de la teoría del capital humano (Becker, 1964; Schultz, 1961) $y$, lo presentado por Fayad \& Easa (2020). Al buscar una justificación para este fenómeno basados en el análisis del mercado laboral y la sociedad colombiana, y en la opinión de expertos tanto del mundo de la empresa como de la academia, aparecen factores de mercado y culturales. Así, por ejemplo, los altos índices de desempleo, que afectan especialmente a los trabajadores más fáciles de reemplazar (escasamente singulares) y que aportan menos valor a la organización, parecen jugar un papel relevante en la decisión de permanecer en la empresa.

En relación a lo anterior, otra posible interpretación podría darse en el hecho de que la mayoría de las empresas de la muestra sean empresas grandes y reputadas, que en su mayoría adoptan prácticas que como consecuencia natural generan el compromiso de los trabajadores al ofrecen una estabilidad en el empleo, prestigio profesional y unas compensaciones por encima de la media, pueden dar luces sobre dicho interés por permanecer en las empresas indistintamente de las $\mathrm{CPCH}$ en las que se vean involucrados los trabajadores.

$\begin{array}{ccc}\text { Desde } & \text { una } & \text { perspectiva } \\ \text { metodológica, } & \text { estos } & \text { resultados } \\ \text { contrarios a lo esperado } & \text { según el }\end{array}$
modelo de configuraciones de practica de Lepak \& Snell (1999-2002) podrían interpretarse, retomando a Boon et al, (2019), al plantear que se presentan problemas en las medidas utilizadas al omitir las sinergias que pueden presentarse entre las diferentes prácticas en la $\mathrm{GCH}$ y los resultados relacionados. Por ello, exponen los autores que no siempre están claro si las configuraciones de práctica como parte del sistema de $\mathrm{GCH}$ es el que causa los efectos en los resultados empresariales. A este respecto, con base en Huo et al, (2016) y Crandall \& Crandall (2008) se podría pensar que las $\mathrm{CPCH}$ en lugar de afectar directamente los resultados financieros, económicos y de productividad, lo hace de manera indirecta a través de su participación en las prácticas organizativas de valor agregado. 
Por último, estos resultados podrían aclararse al retomar a Boon et al, (2019) cuando plantean que la percepción de los empleados sobre las prácticas en la gestión humana de manera particular no son un medio efectivo al momento de identificar los efectos de las $\mathrm{CPCH}$ como subsistema de un sistema organizacional de gestión humana.

\section{Conclusiones}

Teniendo en cuenta las hipótesis formuladas y los resultados alcanzados, se puede concluir que utilizar diferentes $\mathrm{CPCH}$ para distintos grupos de trabajadores puede ser irrelevante para los resultados organizativos contrario a lo planteado por el modelo "ideal" de configuraciones de prácticas de Lepak \& Snell (1999-2002). Por ello y en beneficio de la discusión teórica, se podría pensar que se deben utilizar configuraciones de prácticas de acuerdo con modelos ajustados a las especificidades del ámbito y el entorno de cada organización $\mathrm{y}$, no necesariamente las configuraciones planteadas en el modelo abordado en este estudio.

Ahora bien, para estudios futuros, se debe tener en cuenta, al momento de hacer las medidas, las sinergias que pueden presentarse entre las diferentes prácticas en la $\mathrm{GCH}$ y los resultados relacionados superando con ello la utilización de indicadores aditivos. De igual manera, la sola percepción de los empleados sobre las prácticas particulares y no del sistema completo de $\mathrm{GCH}$, es un medio poco efectivo al momento de identificar los efectos de las $\mathrm{CPCH}$ sobre los resultados organizativos. Se hace necesario abordar el fenómeno desde una perspectiva sistémica en donde se puedan recolectar evidencias institucionales sobre el quehacer en la gestión integral de los mismos.

De otra parte, se debe tener en cuenta para futuras investigaciones, la posibilidad de que las $\mathrm{CPCH}$ impacten los resultados organizativos de manera indirecta mediante su participación en las prácticas organizativas de valor agregado. Se recomienda adoptar mediciones que muestren la relación entre la configuración de prácticas adaptadas a las características del capital humano (valor y singularidad) y, los resultados organizativos mediante variables como la calidad, costos, flexibilidad, agilidad, fiabilidad, propias de la efectividad operativa en relación con la estrategia organizacional. Así mismo se recomienda tener en cuenta factores contextuales (tecnología, cultura, estructura, etc.) como variables mediadoras. Ello, consideramos, permitiría determinar la incidencia directa de las configuraciones de prácticas adaptadas a las características del capital humano en las diferentes organizaciones. Así mismo, el hecho de haber dispuesto de una muestra relativamente pequeña limita la significancia estadística de las relaciones entre variables. De igual manera, el hecho de que dicha muestra la constituyan, en su gran mayoría, empresas multinacionales grandes, puede sesgar su conformación y, estar omitiendo la observación del fenómeno estudiado en empresas que no poseen sistemas de GCH estructurados formalmente.

Por último, es conveniente que en estudios futuros se tenga en consideración la diversidad de sectores a los que podrían pertenecer las empresas que constituyan la muestra $y$, la forma como se valora en sector los malos o buenos resultados de acuerdo a sus propias especificidades. 


\section{Referencias bibliográficas}

Andersén, J. (2019). Resource orchestration of firm-specific human capital and firm performance-the role of collaborative human resource management and entrepreneurial orientation. The International Journal of Human Resource Management, 1-33.

Barney, J. B. (1986). Strategic Factor Markets: Expectations, Luck, and Business Strategy. Management Science, 32(10), 1231-1241.

Barney, J. B. (1991). Firm Resources and Sustained Competitive Advantage. Journal of Management, 17(1), 99120.

Becker, G. S. (1964). Human Capital: A Theoretical and Empirical Analysis, with Special Reference to Education. Nueva York, EEUU: Columbia University Press.

Black, S. E., \& Lynch, L. M. (2004). What's driving the new economy: The benefits of workplace innovation. The Economic Journal, 114(493), F97-F116.

Boon, C., Den Hartog, D. N., \& Lepak, D. P. (2019). A systematic review of human resource management systems and their measurement. Journal of management, 45(6), 24982537.

Buller, P. F., \& McEvoy, G. M. (2012). Strategy, human resource management and performance: Sharpening line of sight. Human resource management review, 22(1), 43-56.

Crandall, R.E., \& Crandall, W.R. (2008). New Methods of Competing in the Global Marketplace. CRC Press, New York.

Delery, J., \& Gupta, N. (2016). Prácticas de gestión de recursos humanos y efectividad organizacional: asuntos de ajuste interno. Revista de eficacia organizativa: personas y rendimiento, 3(2), 139-163.

Dyer, L. (1996). Specialized Supplier Networks as a Source of Competitive Advantage: Evidence from the Auto Industry. Strategic Management Journal, 17, 271-292.

Eisenhardt, K. (1988). Agency- and Institutional-Theory Explanations: The Case of Retail Sales Compensation. Academy of Management Journal, 31(3), 488511.

Fayad, N. M., \& Easa, N. F. (2020). Human resources management practices and employee engagement: known and unknown aspects in the literature. BAU Journal-Creative Sustainable Development, 2(1), 6.

Firth, L., Mellor, D. J., Moore, K. A., \& Loquet, C. (2004). How can managers reduce employee intention to quit? Journal of Managerial Psychology, 19(2), 170-187.

Gorman, C. A., Meriac, J. P., Roch, S. G., Ray, J. L., \& Gamble, J. S. (2017). An exploratory study of current performance management practices: Human resource executives' perspectives. International Journal of Selection and Assessment, 25(2), 193-202.

Hair, Jr., J. F., Hult, G. T. M., Ringle, C. M., \& Sarstedt, M. (2014). A primer on partial least squares structural equation modelling (PLS-SEM). Los Ángeles: Sage Publications.

Huo, B., Ye, Y., Zhao, X., \& Shou, Y. (2016) 'The impact of human capital on supply chain integration and competitive performance. International Journal of Production Economics, 178(1), 132-143. 
Medina Lorza, Aida; Acosta Naranjo, Alejandro; Revuelto Taboada, Lorenzo

Configuraciones de prácticas de recursos humanos y resultados

organizativos

Kehinde, J. S. (2012). Talent Management: Effect on Organizational Performance. Journal of Management Resear HC, 4(2), 7688.

Koch, M. J., \& McGrath, R. G. (1996). Improving Labour Productivity: Human Resource Management Policies Do Matter. Strategic Management Journal, 17, 335-54.

Lepak, D. P., \& Snell, S. A. (1999). The Human Resource Architecture: Toward a Theory of Human Capital Allocation and Development. Academy of Management Review, 24(1), 31-48.

Lepak, D. P., \& Snell, S. A. (2002). Examining the Human Resource Architecture: The Relationship among Human Capital, Employment, and Human Resource Configurations. Journal of Management, 28(4), 517543.

Lepak, D. P., Takeuchi, R. \& Snell, S. A. (2003). Employment Flexibility and Firm Performance: Examining the Interaction Effects of Employment Mode, Environmental Dynamism, and Technological Intensity. Journal of Management, 29(5), 681-703.

Liu, Y., Combs, J. G., Ketchen Jr, D. J., \& Ireland, R. D. (2007). The value of human resource management for organizational performance. Business horizons, 50(6), 503-511.

Mahoney, T. A. (1989). Multiple Pay Contingencies: Strategic Design of Compensation. Human Resource Management, 28(3), 337-347.

Melián-González, S., \& VeranoTacoronte, D. (2008). Estilos de Dirección de RRHH dentro de las Empresas. Cuadernos de Economía y Dirección de Empresas, 11(36), 151-177.
Miles, R. E., \& Snow, C. C. (1984). Designing Strategic Human Resource Systems. Organizational Dynamics, 13(1), 36-52.

Mozo, F., \& Pérez, M. P. (2001). Una Perspectiva Dual para la Gestión de los Recursos Humanos: ¿Optimizar Recursos o Reducir Costes Contractuales? Dirección y Organización, 25, 69-79.

Nonaka, I., \& Takeuchi, H. (1995). The Knowledge-Creating Company. How Japanese Companies Create the Dynamics of Innovation. New York: Oxford University Press.

Pfeffer, J. (1994). Competitive Advantage through People: Unleashing the Power of the Work Force. Boston: Harvard Business School Press.

Pham, H. (2020). Impact of human resource management practices on enterprises' competitive advantages and business performance: Evidence from telecommunication industry. Management Science Letters, 10(4), 721-732.

Quinn, J. B. (1992). Intelligent enterprise. New York: Free Press.

Quinn, J. B., Anderson, P., \& Finkelstein, S. (1996). Managing Professional Intellect: Making the Most of the Best. Harvard Business Review, 74(2), 71-80.

Rousseau, D. M., \& Parks, J. M. (1993). The contracts of individuals and organizations. En L. L. Cummings \& B. M. Staw (Eds.), Research in organizational behaviour (pp. 1-43). Connecticut: Greenwich, JAI Press.

Schultz, T. W. (1961). Investment in Human Capital. American Economic Review, 51(1), 1-17.

Siong, Z. M. B, Mellor, D., Moore, K. A., \& Firth, L. (2006). Predicting Intention to Quit in the Call Center Industry: 
Does the Retail Model Fit? Journal of Managerial Psychology, 21(3), 231 243.

Snell, S. A., \& Dean, J. W. Jr. (1994). Strategic Compensation for Integrated Manufacturing: The Moderating Effects of Job and Organizational Inertia. Academy of Management Journal, 37(5), 11091140.

Tsang, M.C., Rumberger, R.W., \& Levin, H.M. (1991). The Impact of Surplus Schooling on Worker Productivity. Industrial Relations, 30(2), 209-228.

Walsh, C. (1994). Ratios Clave para la Dirección de las Empresas. London: Pitman.
Westwick, C. A. (1997). Manual para la Aplicación de Ratios de Gestión. Bilbao: Ediciones Deusto.

Williamson, O. E. (1975). Markets and hierarchies: Analysis and antitrust implications. New York: Free Press.

Williamson, O. E. (1981). The Economics of Organization: The Transaction Cost Approach. American Journal of Sociology, 87(3), 548-577.

Wuen, C. H., Ibrahim, F., \& Ringim, K. J. (2020). The Impact of Human Resource Management Practices on SMEs Performance: An Exploratory Study in Brunei Darussalam. International Journal of Asian Business and Information Management (IJABIM), 11(2), 68-87. 